\title{
Spectrographs for small telescopes
}

\section{Olivier Thizy and François Cochard}

\author{
Shelyak Instruments, Les Roussets, 38420 Revel, France \\ email: olivier.thizy@shelyak.com, francois.cochard@shelyak.com
}

\begin{abstract}
Shelyak Instruments is a company founded in 2006 offering a full range of spectrographs designed for Astronomy, shipping World Wide. Current users are (1) Public and private observatories who want to setup small telescope for scientific programs or training; (2) Universities for education and demonstrations; (3) Experienced amateurs observers who work in Pro/Amateur collaborations. Lhires III Littrow high resolution spectrograph and eShel optical fibre fed echelle solution are presented with some scientific results including OB stars in which those instruments had a significant contribution.
\end{abstract}

Keywords. instrumentation: spectrographs, stars: emission-line, Be

\section{Introduction}

In 2003, following a CNRS pro/am Astrophysics school in Oleron, several amateurs designed and industrialized a Littrow spectrograph (LHIRES) to reach power of resolution $\mathrm{R}>10000$. Shelyak Instruments now continue the distribution of the Lhires III.

Lhires III is a $F / 10$ Littrow $(F=200 \mathrm{~mm})$ spectrograph with a $15 / 19 / 23 / 35 \mu \mathrm{m}$ slit. Power of resolution $\mathrm{R}$ is around 18000 . Calibration is done with an internal neon lamp.

In 2008, the first commercial optical fiber fed echelle spectrograph - eShel - was available to universities, professional observatories, and amateur astronomers willing to contribute to pro/am collaboration.

Eshel system includes a F/6 Fiber Injection and Guiding Unit, a $50 \mu \mathrm{m}$ optical fiber, a ThAr Calibration Unit and an echelle spectrograph $(125 \mathrm{~mm} \mathrm{~F} / 5$ collimator, R2 echelle grating, cross-dispersing prism, $85 \mathrm{~mm} \mathrm{~F} / 1.8$ objective) with a power of resolution $\mathrm{R}$ above 10000 .

Shelyak Instruments also introduced in 2010 a Low Resolution spectrograph LISA: $\mathrm{F} / 5,23 \mu \mathrm{m}$ slit, $\mathrm{R}=1000$, near infra-red mode.

Figure 1 shows a picture of the Lhires III and eShel spectrographs.

\section{Scientifical Results}

Lhires III main target was Be stars monitoring. An overall pro/am collaboration infrastructure has been put in place: Spectro-L discussion group, ARAS and ARASBeAm web sites, BeSS spectra database, practical workshops... Complemented with the eShel, Lhires III instrument is allowing dozens of amateur around the world to contribute in the Be star monitoring with a continuuous spectroscopic coverage of the bright targets and, as a result, height outbursts discovered in two years and 11000 spectra have been put in the database by amateur astronomers (see ARASBeAm poster, those proceedings).

Other hot stars have been good targets for the Lhires III such as Wolf-Rayet WR140 during 2009 periastron campaign. Hundreds spectra have been taken and the excess emission on top of the CIII 5696 flat line was particularly studied (Fahed et al., these proceedings). 

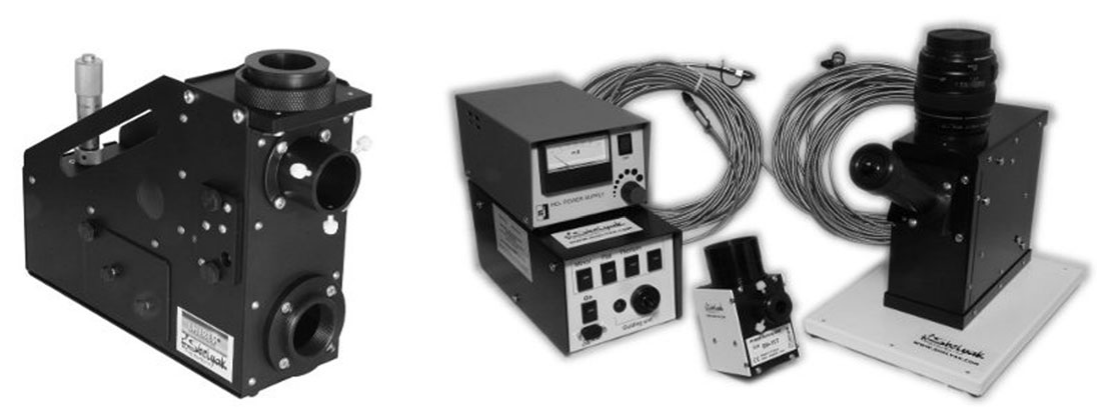

Figure 1. Lhires III and eShel spectrographs

In parallel to WR140 observations, a "Mons campaign" has been put in place to study other OB stars: HD 14134, HD 42087, HD 43384 and HD 52382 supergiants; HD 45314 and HD 60848 Oe stars (Morel et al., these proceedings).

Some pulsating stars have been studied with eShel spectrograph but results have not been published yet: $\beta$ Cep (spectra in BeSS) and BW Vul showing shock waves impact on spectral line shifts.

Luminous Blue Variable P cygni is a target of choice for the Lhires III with studied by Ernst Pollmann and other amateur spectroscopists.

During 2009/2010, an intensive observing campaign has been launched on $\epsilon$ Aur. The transit of the secondary object and its surrounding material is being followed by several amateur astronomers. Robin Leadbeater is focusing on KI 7699 line in particular with great results (Leadbeater \& Stencel 2010). At mid eclipse, more than 100 spectra on KI 7699 line were recorded and around 400 amateur spectra on $\mathrm{H} \alpha$ or sodium D lines.

Several spectroscopic binaries have been studied with Lhires III. But eShel opened the door for much higher radial velocity accuracy studies. It has allowed a group of amateurs (Buil et al., see http://astrosurf.com/buil/extrasolar/obs.htm) to be the first to record four exoplanets with commercially available spectrographs: $\tau$ Boo, HD 189733, HD 195019 and 51 Peg (Buil 2009). An opportunity exists for amateurs to search for new exoplanets around hotter stars or stars with higher rotational speed where the instrument resolution will not limit the radial velocity measurements.

\section{Conclusion}

With off-the-shelf spectrogaphs now widely available, amateur spectroscopy has entered a new era. Professional observatories and universities have now access to affordable standard equipment. Amateur made also some significant contribution to professional programs.

In addition, there is an educational aspect of those instruments with the introduction for several students to high resolution spectroscopy and echelle design.

In the future, more emphasis should be put on spectra archiving and access (extension of BeSS model to other star type) and more structured targeted campaigns (like for WR140 and $\epsilon$ Aur; for exemple $\delta$ Sco periastron in 2011). But it appears now obvious that small telescopes, with more total observing time and flexibility than large telescopes, will contribute to more pro/am collaboration initiatives.

\section{References}

Leadbeater, R. \& Stencel, R. 2010, ArXiv e-prints 1003.3617 